\title{
THREE-DIMENSIONAL SOUND INTENSITY MEASUREMENTS USING MICROFLOWN PARTICLE VELOCITY SENSORS
}

\author{
Hans-Elias de Bree*, W.F. Druyvesteyn**, E. Berenschot*, M. Elwenspoek* \\ * Micromechanical Transducers Group ${ }^{* *}$ Laboratory Signals \& System Network theory \\ University of Twente, P.O. Box 217, 7500 AE Enschede, The Netherlands
}

\begin{abstract}
This paper reports on a novel method to measure three-dimensional sound intensity and the fabrication of a miniature three-dimensional sound intensity probe. Verifying measurements where performed with three separate micro-machined particle velocity probes and one pressure microphone. A three-dimensional sound intensity probe has been realised based on a threedimensional micro-machined particle velocity microphone, a 3D Microflown, and a miniature pressure microphone.
\end{abstract}

\section{INTRODUCTION}

A few years ago I had a discussion with the sales representative of a leading sound \& vibration company. He claimed that the one that was able to make a broadband sound intensity probe smaller than a sugar cube should become a wealthy person. Well, still poor but proud we present the miniature threedimensional sound intensity probe.

Sound intensity is a vector quantity, defined as the time averaged product of the sound pressure (a scalar quantity, p) and the corresponding particle velocity (a vector quantity, u) at the same position. The measurement permits the determination of radiated sound power without requiring special acoustic environments such as reverberation and anechoic chambers. The measurement technique is also used for sound source localisation.

Since historically no particle velocity probes were available, the traditional way to measure sound intensity was to use two closely spaced and phase matched pressure microphones. The particle velocity is calculated by the difference of the signals from both the pressure microphones and the (average) sound pressure is found from the sum of both. This method is called the p-p method, see Fig. 1 [1].

Two major disadvantages of the p-p probe are its high prize and the fact that performing broad band measurements is time-consuming. Furthermore the bandwidth of the probe is limited and the probe is fragile.

Nowadays the Microflown [2] can perform the particle velocity measurement directly and a sound intensity measurement becomes possible without the mentioned disadvantages. A Microflown can be considered as a mass flow sensor that has been optimised for sound measurements. It consists of two very closely spaced $(40 \mu \mathrm{m}$ apart) heated platinum resistive temperature sensors. The temperature difference of both sensors is linearly dependent on the particle velocity. In combination with a pressure microphone a Microflown can form a sound intensity probe, a so called p-u method, see Fig. 2 [3].

A one dimensional sound intensity probe can be extended to a three dimensional probe by using three $p-p$ probes (six microphones) or one microphone and three Microflowns ( $\left.p-\mathbf{u}^{3}\right)$. The realisation of a three-dimensional $p-p$ probe however is not very realistic. The prize of such probe will be over 30,000 USD and the frequency range is limited even more than the $1 \mathrm{D} p-p$ probe.

$A$ broad band three-dimensional sound intensity probe however will offer many advantages. The magnitude and direction of the acoustic energy flow can be measured directly, this will improve the effectiveness of the measurement. The 3D measurement allows measuring non-stationary sound fields as for example the noise of moving objects like aeroplanes.

Using of Microflown technology, a threedimensional sound intensity probe become feasible.

Preliminary measurements with three separate (half-inch packaged) Microflowns and a half-inch pressure probe showed promising results.

Now a fully integrated three-dimensional Microflown has been realised. This will be another step in the direction of realisation of a rugged broad band three-dimensional sound intensity probe. 


\section{SOUND INTENSITY}

Sound intensity is a vector quantity that describes the amount and direction of net flow of acoustic energy at a given position. It is defined as the time averaged product of the sound pressure (a scalar quantity, $p$ ) and the corresponding particle velocity (a vector quantity, $\mathbf{u}$ ) at the same position.

$$
I=\frac{1}{T} \int p(t) \cdot u(t) d t
$$

The electrical analogy is well known: power $=$ voltage $\times$ current.

Two methods to measure the sound intensity will be explained briefly: the $p-p$ probe and the p-u probe. For simplicity first the two methods will be compared in their one directional realisation. The pros and cons will be extended later to the three dimensional versions.

\section{The P-P PIROBE}

A p-p probe consists of two, closely spaced, matched pressure microphones [1].

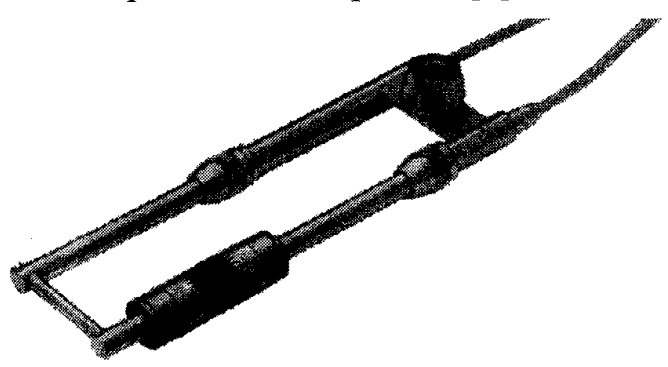

Fig. 1: $1 D p-p \quad B \& K$ sound intensity probe (The microphones are a half inch in diameter).

The particle velocity is calculated by the subtraction of the signals from both pressure microphones. The method is based upon Newton's second law, the equation of motion:

$$
\rho \cdot \frac{\partial u_{r}}{\partial t}=-\frac{\partial p}{\partial r}
$$

Where $\rho$ is the density of the medium, $u_{r}$ is the particle velocity in one direction $r$, and $p$ the instantaneous pressure. Since the pressure gradient is proportional to the particle acceleration, the particle velocity can be obtained by integrating the pressure gradient in respect to time.

$$
u_{r}=-\frac{1}{\rho} \int \frac{\partial p}{\partial r} d t
$$

By measuring the pressure at two points at a close distance $\Delta r$, the pressure gradient is approximated.

$$
u_{r}=-\frac{1}{\rho \Delta r} \int\left(p_{a}-p_{b}\right) d t
$$

This approximation is valid as long as the separation, $\Delta r$, is small compared to the wavelength.

\section{BANDWIDTH (P-P)}

The proper determination of the particle velocity is frequency limited. The upper frequency is limited due to the fact that the estimation $\left(\partial p / \partial r=\left(p_{a}-p_{b}\right) / \Delta r\right)$ no longer holds. The lower frequency limit is caused by the phase mismatch of the two microphones and electronics.

In practice this means that the lowest frequency that can be measured is best case (anechoic conditions) $20 \mathrm{~Hz}$ and worst case $350 \mathrm{~Hz}$ (reverberation, phase mismatch $=0.3^{\circ}$ ). The smallest size of the microphone separation equals the microphone diameter and bounds the highest frequency that can be measured (approximately 10kHz).

\section{SPACERS (P-P)}

To measure the complete bandwidth with a p-p probe three spacers and two types of microphones are used. Two half inch microphones and a spacer of $50 \mathrm{~mm}$ are used for the lower frequencies $(20 \sim 350 \mathrm{~Hz}$ to $1250 \mathrm{~Hz})$ the $12 \mathrm{~mm}$ spacer is used for the medium frequencies $(250 \sim 1250$ to $5 \mathrm{kHz})$. Two quarter inch microphones and a spacer of $6 \mathrm{~mm}$ are used to measure in the higher frequencies $(250 \sim 2000$ to $10 \mathrm{kHz})$. The $\mathrm{p}-\mathrm{p}$ probe has to be disassembled and re-calibrated when changing spacers or microphones. This makes the broad band sound intensity measurement time consuming furthermore the use of these spacers will make the realisation of the probe fragile.

\section{NEARFIELD MEASUREMENTS (P-P)}

When a $p-p$ probe is positioned in the nearfield of a sound source, the sound intensity is not constant from one pressure probe to the other. This will lead to an overestimation. This effect is present when the probe is placed closer to the source than at least two times the separation (of the microphones) and can be a problem at low frequencies. 


\section{SIGNAL TO NOISE RATIO (P-P)}

An example of a high quality matched pair is the Brüel \& Kjær sound intensity microphone pair B\&K 4181 [5]. One of the $1 / 2$ inch matched microphones has a selfnoise of $20 \mathrm{~dB}(\mathrm{~A})$ and a sensitivity of $11.2 \mathrm{mV} / \mathrm{Pa}$ (at $1 \mathrm{kHz})$.

It will be convenient to express noise values in voltages and in one Hertz bandwidth. The noise is expressed in $\mathrm{V} / \sqrt{\mathrm{Hz}}$.

The latter corresponds to: $U_{\text {noise }}=31.6 \mathrm{nV} / \sqrt{\mathrm{Hz}}$ at $1 \mathrm{kHz}$ [5]. ( $U_{\text {signal }}=11.2 \mathrm{mV}$ at $1 \mathrm{~Pa}(=94 \mathrm{~dB} \mathrm{SPL})$ pressure at $1 \mathrm{kHz}$ ). The microphones do have a $1 / \mathrm{f}$ noise behaviour that will be dominant at frequencies under $500 \mathrm{~Hz}$ [8]. Because the differential pressure is measured to determine the particle velocity, two microphones are used, the total noise will increase by a factor $\sqrt{2}$. This is depicted in Fig. 2. A plane wave with a sound intensity level of $94 \mathrm{~dB}$ is offered to the $p-p$ probe. The signal (that will be used to calculate the particle velocity) is produced by the subtraction of both pressure microphone outputs.

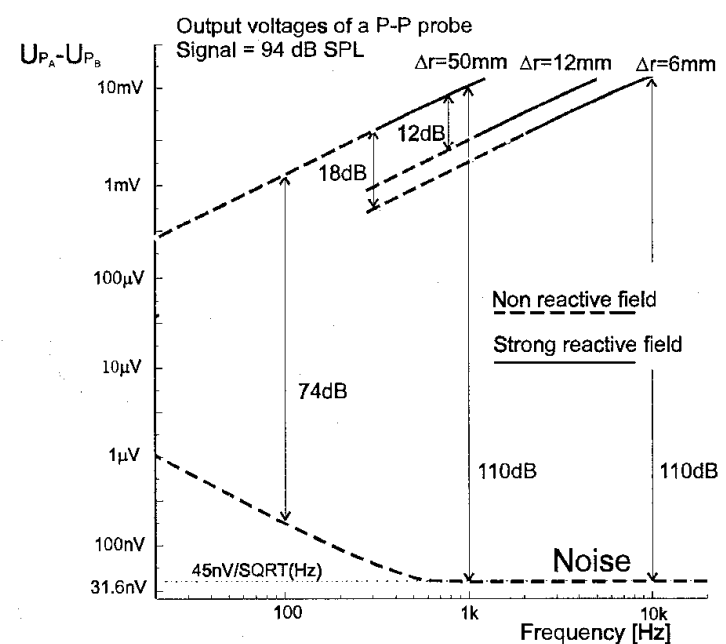

Fig. 2: Noise output voltages in $\mathrm{V} / \mathrm{V} / \mathrm{Hz}$ and signals due to $94 d B$ SIL

As can be seen in Fig 2, when the frequency decreases the signal decreases and the noise increases; the signal to noise ratio decreases rapidly for frequencies lower than $500 \mathrm{~Hz}$. The signal will be integrated, see eq. (3) and therefore the frequency response will be flat, the noise however will also be integrated: the signal to noise ratio therefore will not be altered.

\section{The P-U Probe}

Historically there has been a lack of particle velocity probes. Nowadays the Microflown [2] can perform the particle velocity measurement directly and a p-u probe can be realised [3]. The probe consists of a pressure microphone and a particle velocity microphone.

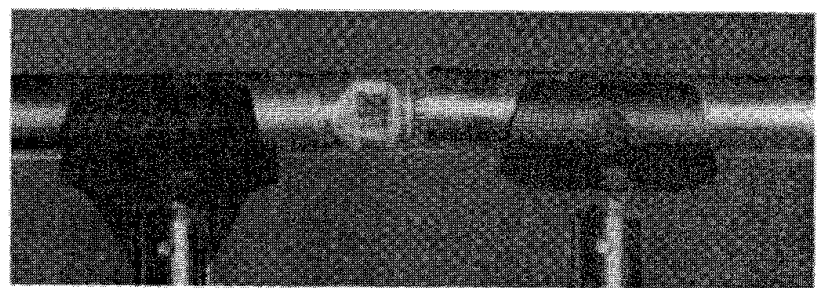

Fig. 3: $1 D p-u$ sound intensity probe (The microphone and Microflown are a half inch in diameter).

The Microflown has a first order frequency behaviour. For low frequencies $(f<300 \mathrm{~Hz})$ the frequency response will be flat and the phase shift will be zero degrees. For higher frequencies the $(f>3 \mathrm{kHz})$ the frequency response will be exactly $-6 \mathrm{~dB} /$ oct and the phase shift will be -90 degrees. At its corner frequency the Microflown has a $-3 \mathrm{~dB}$ response and a phase shift of -45 degrees.

It is not very difficult to correct the frequency behaviour of the Microflown electronically.

\section{BANDWIDTH (P-U)}

The bandwidth of a Microflown and a pressure microphone can not be compared easily. Above the corner frequency a pressure microphone can not be used. The Microflown however has an exact first order frequency behaviour. Therefore the Microflown can be used above its corner frequency. The present value of the corner frequency is $1 \mathrm{kHz}$.

For sound intensity purposes the lowest usable frequency will be determined by the pressure microphone and can be found lower than $1 \mathrm{~Hz}$.

It is hard to give a distinct high frequency limit of a $\mathrm{p}$-u probe. The signal to noise ratio will drop $6 \mathrm{~dB} / \mathrm{oct}$ as the frequency rises above the corner frequency. With the existing Microflowns the signal to noise ratio $(\mathrm{S} / \mathrm{N})$ is less in the $1 \mathrm{kHz}$ to $10 \mathrm{kHz}$ bandwidth; above the $10 \mathrm{kHz}$ the p-p probe can not be used so the p-u probe will perform better.

With the averaging time kept constant the $\mathrm{S} / \mathrm{N}$ of the $\mathrm{p}-\mathbf{u}$ sound intensity probe will however drop not the expected $6 \mathrm{~dB} /$ oct but $3 \mathrm{~dB} /$ oct above the corner frequency of the 
Microflown. The averaging of the product of pressure and particle velocity causes this effect.

\section{NEARFIELD MEASUREMENTS (P-U)}

The $\mathrm{p}-\mathrm{u}$ probe measures the sound intensity at one place and therefore the sound intensity will not alter along the probe and no overestimation will be noticed.

\section{DISCRETISATION ERROR (P-U)}

Since the Microflown and microphone are not situated exactly on the same place, a phase shift can occur. This can cause an estimation error that will be smaller than $1 \mathrm{~dB}$ at frequencies below $5 \mathrm{kHz}$.

\section{SigNAL TO NOISE RATIO (P-U)}

To calculate the signal to noise ratio a practical example of a Microflown will be examined. The sensitivity of the Microflown is defined as the differential resistance variation of both sensors and is measured at $94 \mathrm{~dB}$ PVL at $100 \mathrm{~Hz}$. The two temperature sensors (platinum resistors) of the Microflown have to be heated with a total nominal power of $100 \mathrm{~mW}$. The sensitivity $(\Delta \mathrm{R} / \mathrm{R}$ at $94 \mathrm{~dB}$ SPL $)$ is then on the order of $0.3 \%$.

$$
U_{o u t}=I R \frac{\Delta R}{R} \sqrt{\frac{f_{c}^{2}}{f_{c}^{2}+f^{2}}}
$$

Using $f_{c}$ as the corner frequency of the Microflown $\left(f_{c} \approx 1 \mathrm{kHz}\right)$. The Microflown is operated at nominal power $(\mathrm{I}=6.3 \mathrm{~mA}$, $\mathrm{R}=1300 \Omega$ ). The output voltage for frequencies below the corner frequency can be calculated by:

$$
U_{\text {out }}=I R \frac{\Delta R}{R}=2.5 \mathrm{mV}
$$

For frequencies above the corner frequency the output voltage will decrease $6 \mathrm{~dB} /$ oct.

The output noise of the Microflown is caused by thermal noise only and is given by eq. (6).

$$
U_{n o i s e}=\sqrt{2 k T_{s} R}=5 n V / \sqrt{H z}
$$

Using $T_{s}$ as the absolute mean temperature of the sensor $\left(\mathrm{T}_{\mathrm{s}} \approx 700 \mathrm{~K}\right), R$ as the resistance $(R \approx 1300 \Omega)$ of one sensor and $k$ as Boltzmann's constant $\left(1.38 \times 10^{-23} \mathrm{~J} / \mathrm{K}\right)$. In the acoustic bandwidth $1 /$ f-behaviour is not observed. The previous is summarised in Fig. 4.

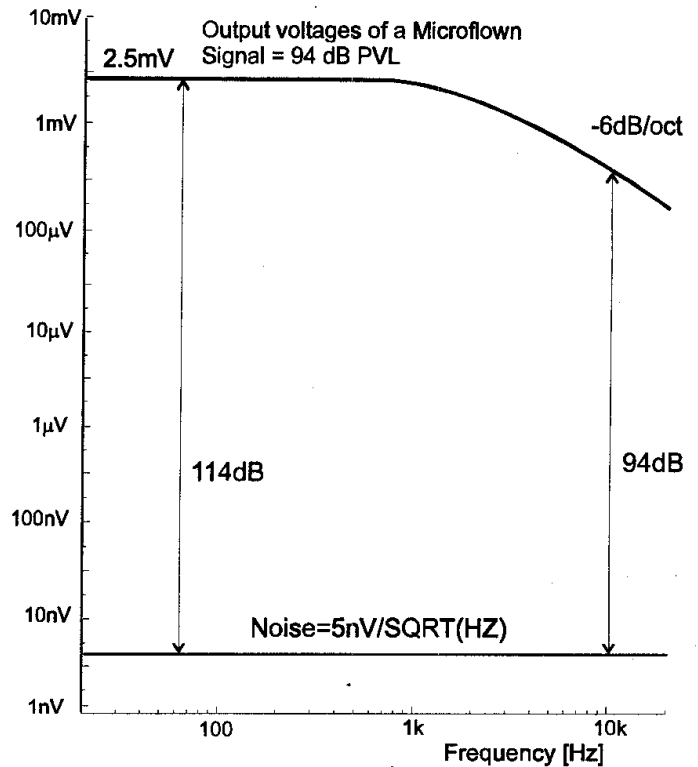

Fig. 4: Noise output in $\mathrm{V} / \sqrt{\mathrm{Hz}}$ and signals due to 94dB SIL.

\section{D PROBES}

As mentioned the realisation of a 3D p-p probe is not very practical. The result will be a very expensive and large probe, see Fig. 5. Measuring high frequencies is limited by the minimal spacing and can be expected maximal $3 \mathrm{kHz}$.

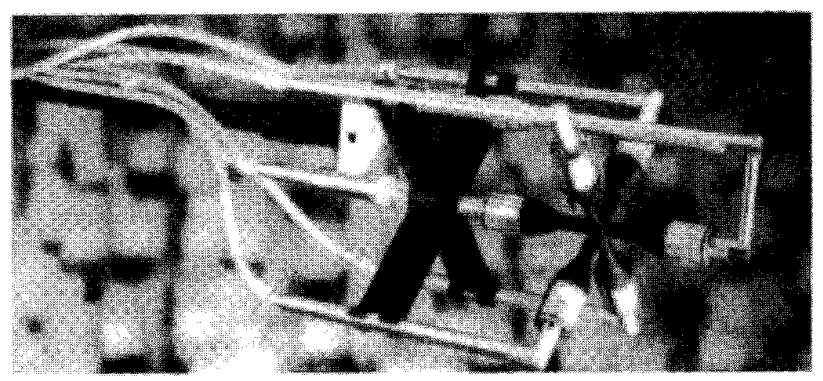

Fig. 5: $3 D B \& K$ p-p sound intensity probe (The microphones are a half inch in diameter).

In principle the realisation of a three dimensional Microflown will not triple the costs and the realisation can be kept relatively small.

Initially the signal to noise ratio of the $\mathrm{p}-\mathrm{u}^{3}$ probe will be several $d B$ less than the $S / N$ of the one-dimensional $p$-u probe. The less optimal packaging of this first realisation causes this effect. Further research is needed to optimise the probe to the quality of the 1D version. 


\section{COMPARING BOTH METHODS}

Comparing both methods first one has to realise that the $p-p$ probe is the world standard. All laws, standards and guidelines are drafted for $p-p$ probes. Therefore one will consider using a p-u only when its overall performance is much better than the existing probes.

For a p-p probe the phase error between the two microphones has to be less than $0.3^{\circ}$. Even then measuring low frequencies $(\mathrm{f}<100 \mathrm{~Hz})$ is very difficult. Measuring a low-level low-noise source (such as for example a refrigerator) is practically impossible nowadays with a p-p probe [4].

The $\mathrm{p}-\mathbf{u}$ probe can be constructed more rugged than a $p-p$ probe since various spacers and microphones are not required.

The broad band measuring time is much lower if one uses a p-u probe. No spacers or microphones have to be altered and the probe has therefore to be calibrated only once. For low frequencies the signal to noise ratio of the p-u probe is much better. So less averaging time is required to get a consistent result.

For frequencies higher than $1 \mathrm{kHz}$ the $\mathrm{p}-\mathrm{p}$ probe performs better nowadays. The p-p probe however has to change spacers in the $1 \mathrm{Khz}-$ $10 \mathrm{kHz}$ frequency band and measuring higher frequencies than $10 \mathrm{kHz}$ becomes difficult. In principle the $\mathrm{p}-\mathbf{u}$ probe can measure higher frequencies than $10 \mathrm{kHz}$ but the $\mathrm{S} / \mathrm{N}$ will not be very high. A poor low frequency $\mathrm{S} / \mathrm{N}$ is worse than a poor high frequency $S / N$ since the $S / N$ decreases linear with the square root of the number of averages: Hundred-thousand averages at $10 \mathrm{kHz}$ will take only ten seconds at 10 Hertz it will take 2.8 hours!

At last, the topic of this article: with the Microflown technology it is relatively easy to fabricate a low-cost high-bandwidth $3 \mathrm{D}$ sound intensity probe with very small dimensions.

\section{MEASUREMENTS}

There are two contrasting standard acoustic measurement set-ups: The anechoic and the reverberation chamber. In the first no acoustic reflections are present. In such environment one is able to perform free field measurements.

The reverberation chamber is quite the opposite: the walls of this chamber will reflect almost all sounds. In such environment diffuse plus direct sound-fields can be generated.

The sound intensity probe measures the sound energy flow, therefore a well performing sound energy probe should produce the same results in both the anechoic as the reverberation room.

Only the net flow of acoustic energy is measured since in an anechoic chamber no reflections are present. A sound intensity probe is supposed to measure only this net flow. So if the acoustic measurement is repeated in a reverberation chamber, the result must be the same: the total effect of the diffuse field will be zero and only the direct sound field is measured, see Fig 6.
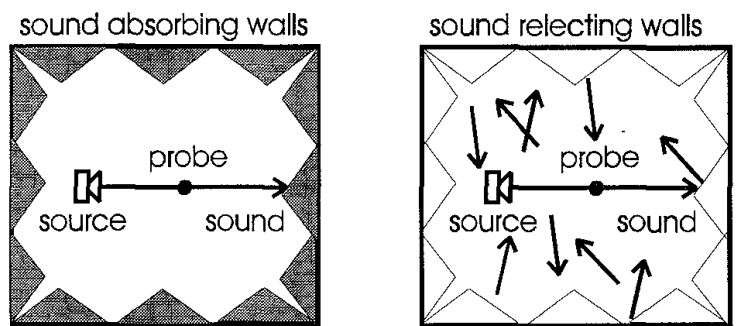

Fig. 6: Two measurement set-ups. Left the anechoic room: only a direct sound field. Right the reverberation room: a direct and a diffuse sound field is generated.

One basic verifying measurement is shown. Measured at $80 \mathrm{~dB}$ SIL in anechoic and reverberation chamber and the difference in output of both are shown. If $p-\mathbf{u}^{\mathbf{3}}$ probe was positioned in $[0,0,0]$, the sound source is placed at $[0.57 \mathrm{~m}, 0.57 \mathrm{~m}, 0.57 \mathrm{~m}]$.

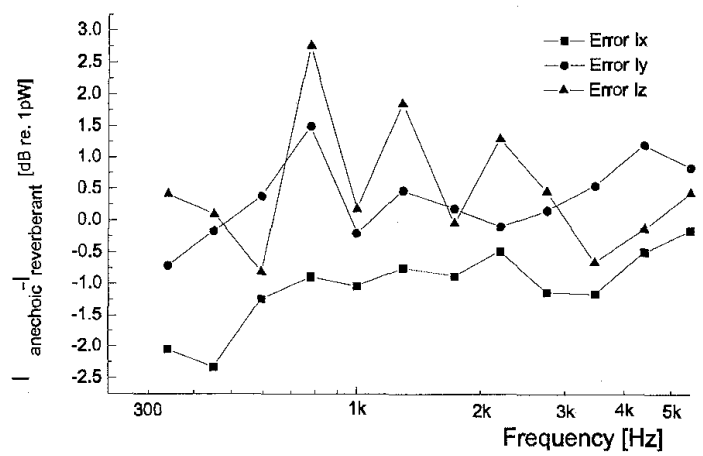

Fig. 7: Verifying 3D sound intensity Measurements.

As can be seen in Fig 7 the 3D sound intensity $\mathbf{I}$ is measured with a maximum error of approximately $3 \mathrm{~dB}$.

The overall under estimation of $I_{x}$ could be caused by the measurement set up (the intensity was poined to the floor) and the error in $I_{z}$ could be caused by the $90^{\circ}$ angle of the microphone and Microflown. More measurements have to be performed to find the source of these errors. 


\section{REALISATION OF THE P-U $\mathrm{U}^{3}$ PROBE}

The first step in the realisation of the $p-\mathbf{u}^{3}$ probe is the micromachining part. The special part is solving the paradox of realising a three dimensional probe in the two dimensional (planar) technique.

The through-the-wafer etching solves the planar character: $\mathbf{u}_{\mathbf{y}}$ and $\mathbf{u}_{\mathrm{z}}$ Microflows are made on the surface and the $\mathbf{u}_{x}$ Microflown is obtained through the wafer, see Fig. 5.

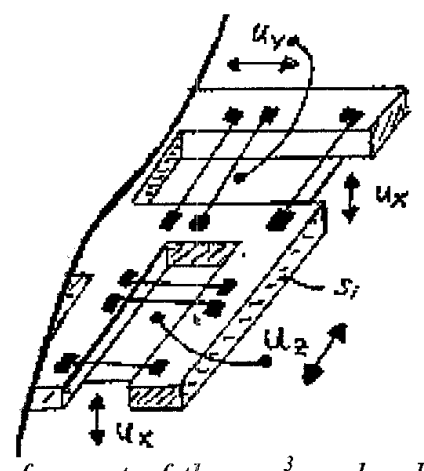

Fig. 3: sketch of a part of the p-u probe showing how the three; orthogonal measuring directions are positioned.

A $300 \mathrm{~nm}$ low-stress silicon nitride layer is deposited on a 3" silicon wafer by low-pressure chemical vapour deposition. A $200 \mathrm{~nm}$ platinum / $10 \mathrm{~nm}$ chromium layer is deposited and patterned on the bottom and on top of the wafer. The chromium layer is used for adhesion purposes.

The silicon nitride layer is patterned (top and bottom) with a reactive ion etching technique. The finalising step of the micro machining is the anisotropic wet chemical etching with a $\mathrm{KOH}$ solution.

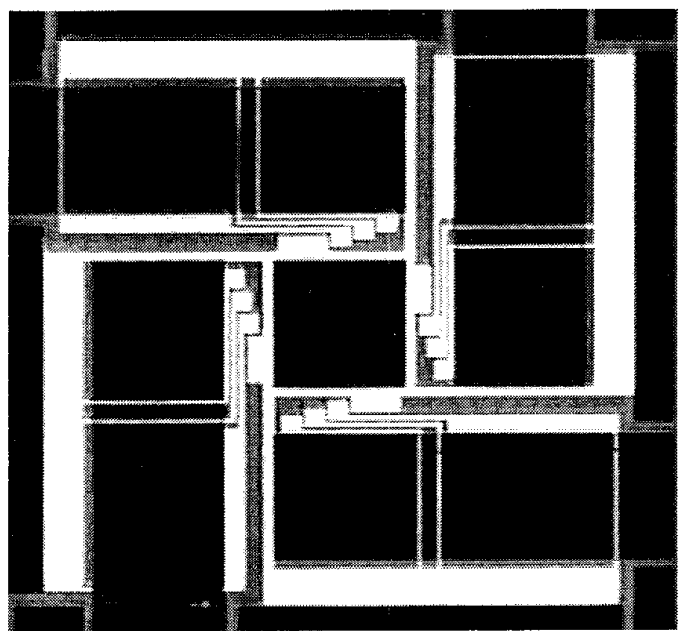

Fig. 6: Micro machined 3D particle velocity sensor (half inch square).

\section{Conclusion}

It is possible to measure sound intensity in three dimensions with the use of three Microflowns and a pressure microphone.

Measurements showed satisfying results up to $5 \mathrm{kHz}$ with a realisation consisting of three half-inch Microflowns and a half-inch pressure microphone.

A three dimensional Microflown has been realised and with this sensor it will be possible to construct a miniature three-dimensional sound intensity probe.

The novel method has a better signal to noise ratio for low frequencies $(\mathrm{f}<100 \mathrm{~Hz})$. It performs equally good for frequencies between $100 \mathrm{~Hz}$ and $1 \mathrm{kHz}$. For frequencies above the $1 \mathrm{kHz}$ the probe has a lower signal to noise ratio.

The novel method allows to measure the complete audio band at once.

\section{REFERENCES}

[1] F.J. Fahy, Sound Intensity, second edition, E\&FN SPON, London, 1995

[2] H.E. de Bree et al, The Microflown, Sensors and Actuators: A, Physical, volume SNA054/1-3, pp 552$557,1996$.

[3] W.F. Druyvesteyn et al, A new sound intensity probe; comparison to the Bruel \& Kjaer p-p probe, 104th AES Convention Amsterdam; pre-print 4651, 1998

[4] F. Jacobsen, Sound intensity measurement at low levels, Journal of Sound and Vibration, 1993

[5] Brüel \& Kjær, Sound Intensity, Technical Review, No. 3, 1982.

[6] Brüel \& Kjær, Product Data, Sound Intensity Microphone Pairs - Types 4178 4181, 1995.

[7] Master Catalogue, Electronic Instruments, Brüel \& Kjær, may 1989.

[8] G.S.K. Wong, T.F.W. Embleton, AIP Handbook of condenser microphones, Theory, calibration, and measurements, 1994, New York. 